\title{
Regulación de la enfermería en México: actores, procesos y resultados
}

\author{
Regulation of nursing in Mexico: actors, processes and outcomes
}

Patricia Aristizabal (https://orcid.org/0000-0001-5501-239X) ${ }^{1}$

Gustavo Nigenda (https://orcid.org/0000-0003-0067-0664) ${ }^{2}$

Allison Squires (https://orcid.org/0000-0002-5238-2122) ${ }^{3}$

Yetzi Rosales (http://orcid.org/0000-0003-1169-9411) ${ }^{4}$

${ }^{1}$ Facultad de Estudios Superiores Iztacala.

Universidad Nacional Autónoma de México. Av. De los Barrios 1, Hab. Los Reyes Iztacala, Barrio de los Arboles / Barrio de los Héroes. 54090 Tlalnepantla de Baz Edo. México México.

aristizabalhpatricia@ gmail.com

${ }^{2}$ Escuela Nacional de Enfermería y Obstetricia. Universidad Nacional Autónoma de México. Ciudad de México México. ${ }^{3}$ NYU Rory Meyers College of Nursing. Nueva York NY EUA.

${ }^{4}$ El Colegio de la Frontera

Norte. Tijuana BC México.
Abstract This paper aims to analyze the regulatory structure of nursing in Mexico through its legislation and perspectives of participant stakeholders. A case study was undertaken using qualitative and quantitative data sources, as well as from the review of official documents. The anal$y$ sis included data from the sources according to the four realms proposed by the Moran \& Wood (1993) model. The more advanced realm was the market entry since the state regulates entry through a license of practice. The regulation of competition is weak, showing very reduced areas of autonomous practice. The labor market is offering a wide variety of entry options with clear signs of deterioration. The dominant payment mechanism is salary, showing a structure that incorporates a component that does not impact on pensions at the end of the labor cycle. The regulation of nursing is a component of its professionalization, and as such, it is understood as a multidimensional consolidating process, particularly realms related to the regulation of competition, market structure, and payment mechanisms, in which nursing representatives should play a more active role in the future.

Key words Nursing, Legislation, Mexico, Health workforce, Health care sector
Resumen El objetivo de este artículo es analizar la estructura regulatoria de la enfermería en México, a través de la normatividad y los actores involucrados. Se desarrolló un estudio de caso con uso de fuentes de información cualitativa, cuantitativa y revisión de documentación oficial. El análisis integró los datos obtenidos de las fuentes de acuerdo con cuatro dimensiones propuestas por Moran \& Wood (1993). La dimensión con mayores avances es el ingreso al mercado ya que el Estado regula el ingreso a través de una licencia de práctica. La regulación de la competencia es débil y muestra áreas aisladas de práctica autónoma. El mercado laboral ofrece condiciones variadas de inserción con muestras claras de deterioro. El mecanismo de pago dominante es el salario de las instituciones públicas mostrando una estructura que incorpora un componente que no cotiza para la pensión al término del ciclo laboral. La regulación de la enfermería es un componente de su profesionalización y como tal se distingue como un proceso en marcha en vías de consolidación, particularmente aquellas dimensiones relacionadas con la regulación de la competencia, la estructura del mercado y los mecanismos de pago en el cual la enfermería y sus representantes deben jugar un papel más activo.

Palabras clave Enfermería, Legislación, México, Fuerza laboral de salud, Sector salud 


\section{Introducción}

La enfermería en México atraviesa por importantes cambios. Por un lado se observa el incremento de años y niveles de entrenamiento, y por otro, un mercado laboral dinámico pero con señales de deterioro ${ }^{1}$.

Aunque las enfermeras con nivel licenciatura se entrenan desde hace más de medio siglo, es apenas en los últimos 10 años cuando el volumen de producción se ha incrementado exponencialmente. El mercado laboral empieza a mostrar interés por reclutar enfermeras licenciadas en detrimento de las técnicas en enfermería que por décadas dominaron la oferta ${ }^{2}$. En la formación, el incremento de la participación privada es particularmente relevante. En otros países se ha observado un aumento importante del número de escuelas privadas que entrenan enfermeras de nivel técnico y de licenciatura. El entrenamiento privado se hace desde una lógica de negocio con dos vertientes, una de baja y otra de alta calidad, siendo la primera la más prevalente. México sigue este patrón el cual también se ha observado en países como Brasil y Colombia ${ }^{3}$.

El deterioro del mercado laboral también está documentado. Desde hace una década se reportaron tasas de subempleo y desempleo entre las enfermeras mexicanas egresadas de universidades $^{1}$ a pesar de que los indicadores de personal de enfermería por población en el país mostraban la necesidad de formar más enfermeras. Estos dos fenómenos del mercado, denotaban la clara necesidad de una intervención estatal para incrementar la demanda de este personal por parte de las instituciones de salud. En la actualidad, un fenómeno del mercado que ha sido reportado ya en países como Brasil es la precarización ${ }^{4}$. En México este fenómeno no ha sido analizado para la enfermería y sin embargo, existen condiciones que parecerían estarlo promoviendo como el aumento de contratos temporales y la tercerización de las relaciones laborales.

Por otra parte, es importante considerar que la enfermería en México se encuentra en un proceso de profesionalización ${ }^{5}$ a través del cual busca incrementar sus años de entrenamiento para adquirir conocimientos más específicos sobre la atención a la salud de la población e intentar establecer un monopolio de práctica ${ }^{6}$.

La regulación profesional puede ser entendida, en general, como un "marco dinámico dentro del cual se pueden identificar las normas profesionales para servir a la protección pública. Los sistemas regulatorios deben ser enfocados, flexi- bles y habilitadores, asegurando que los estándares sean claros, visibles y alcanzables"7.

El desarrollo de la regulación de la enfermería en México, se contextualiza a partir de la existencia de factores como las reformas de salud, los cambios demográficos y epidemiológicos, las nuevas modalidades de atención, así como el ejercicio de los derechos laborales ${ }^{8}$.

\section{El modelo de estructura de regulación profesional}

La participación de los profesionales en la producción de servicios de salud puede ser estudiada a través del modelo de regulación propuesto por Moran \& Wood", definido como "el mecanismo utilizado para establecer las características de la práctica y los estándares técnicos que ésta tiene que alcanzar". Este modelo incluye cuatro dimensiones: 1) mecanismos de ingreso al mercado; 2) control de la competencia profesional; 3) estructura del mercado de trabajo; y 4) mecanismos de pago $^{10}$.

El mecanismo del ingreso al mercado hace referencia a las condiciones requeridas para practicar o ejercer la enfermería. El objetivo es la obtención de un certificado o licencia profesional después de cumplir con los requisitos de escolaridad que acrediten al portador como persona idónea para ejercer dicho cargo.

La regulación de prácticas de competencia está relacionada con funciones compartidas entre grupos ocupacionales del campo de la salud o con funciones no establecidas claramente o de reciente creación y que pueden ser objeto de duplicidad entre el personal de salud. Esta regulación implica el "cierre" ${ }^{11}$ que los miembros de una profesión llevan a cabo para evitar o limitar la participación de otros grupos en las actividades que esa profesión ha definido como su área de influencia.

La estructura del mercado de trabajo, se relaciona con la forma en que los grupos profesionales se insertan en el mercado laboral. En la gran mayoría de países, la forma más común en la que el personal de enfermería se vincula al mercado de trabajo es a través de instituciones de salud públicas y con mecanismos de asalariamiento? Es aún escaso el desarrollo de ejercicio independiente y de la producción de servicios privados, fuera del ambiente institucional.

El objetivo de este trabajo es analizar la estructura regulatoria de la enfermería en México, a través de la normatividad y los actores involucrados. 


\section{Metodología}

El método utilizado fue el "estudio de caso"12. La presentación de resultados deriva del uso de datos cualitativos complementados con datos cuantitativos. Dicho estudio se realizó en tres fases simultáneas que se describen a continuación.

La primera fase consistió en una revisión documental que incluyó: noticias, leyes, herramientas normativas, metodológicas y legales acerca de la regulación del ejercicio de la enfermería en México. Se incluyeron documentos publicados entre los años 2005 y 2018 que contenían información acerca de la reglamentación mencionada.

La segunda fase incluyó datos secundarios de la Encuesta Nacional de Ocupación y Empleo (ENOE, 2018) y del Sistema Información Administrativa de Recursos Humanos en Enfermería (SIARHE, 2018). Esta información se consideró para ofrecer un contexto cuantitativo del fenómeno de estudio. La ENOE tiene un diseño probabilístico representativo a nivel nacional, es aplicada por el Instituto Nacional de Estadística y Geografía (INEGI ${ }^{13}$ y se encuentra disponible en su sitio web (https://www.inegi.org.mx/programas/enoe/15ymas/). La información obtenida del SIARHE se deriva de una encuesta no probabilística que realiza la Secretaría de Salud anualmente para recabar datos de la situación laboral del personal de enfermería del país. Se encuentra disponible en: http://www.salud.gob.mx/unidades/cie/siarhe/.

En la tercera fase se realizaron entrevistas (entre mayo de 2018 y febrero de 2019) a personal de enfermería que en ese momento laboraba en puestos de decisión gubernamentales con funciones de planificación y gestión de los recursos humanos, tanto del sector salud como del sector educativo; además se entrevistó a enfermeras que ejercían roles clínicos, comunitarios, educativos, investigativos y administrativos y que tenían como mínimo cinco años de experiencia laboral. Todas laboraban en instituciones públicas.

Se realizaron 18 entrevistas (13 mujeres y 5 hombres). Todos los participantes otorgaron su consentimiento ${ }^{14}$. Éstos fueron contactados y entrevistados en la Ciudad de México. Dado el nivel de información y de experiencia así como de su perspectiva en la estructura institucional, sus opiniones ayudaron en la aproximación al fenómeno en escala nacional.

El acceso a los informantes se realizó a través de "porteros" 15 y la selección de los sujetos a través de un "muestreo de máxima variación", que en este caso, correspondía al personal de salud y enfermeras/os que cumplían con las características descritas anteriormente. Las entrevistas fueron procesadas a través de un análisis de contenido ${ }^{16}$. Se resguardó la identidad de los participantes asignando códigos a cada entrevista.

Este trabajo se deriva de una investigación más amplia sobre la profesionalización de la enfermería en México, la cual fue evaluada por el comité de ética del Instituto Nacional de Salud Pública de México, previo a la recolección de datos primarios, con lo cual se valida el cumplimiento de los aspectos éticos de la misma.

\section{Resultados}

Los resultados de las cuatro dimensiones propuestas por Moran y Wood se ordenan en tres apartados: los actores participantes en el proceso, los reglamentos o procesos existentes definidos por la autoridad correspondiente y los resultados del ejercicio regulatorio obtenidos entre 20052018.

El Cuadro 1 presenta los documentos orientados hacia la regulación de la enfermería en México. Estos documentos son la base para el análisis de los actores que participan en la regulación, en los procesos y en los resultados de cada dimensión.

\section{Mecanismos de ingreso al mercado}

Los actores presentes en este proceso regulatorio son: a) el Congreso de la República al establecer como requisito la obtención de un título profesional, b) las Instituciones de Educación Superior (IES) al emitir dicho título y c) la Dirección General de Profesiones (DGP) de la Secretaría de Educación Pública, al emitir una cédula profesional al nuevo egresado, equivalente a una licencia de práctica.

La DGP emite la licencia de práctica, sin embargo, la intervención del Estado por hacer cumplir la regulación es muy limitada dado que es frecuente que los estudiantes de la licenciatura logren insertarse laboralmente en unidades de salud, principalmente privadas. Las instituciones públicas sí solicitan la licencia de práctica para contratar una enfermera, incluso de tiempo parcial.

Una nueva estrategia de re-ingreso a un mercado especializado es a través de la certificación de profesionales. Esta consiste en la comprobación de conocimientos teóricos en un área específica, que los convierte en profesionales idóneos 
Cuadro 1. Documentos orientados hacia la regulación de la enfermería en México.

\begin{tabular}{|c|c|c|c|}
\hline Actores & Ley & Cap./ Art./ Año & Contenido \\
\hline Congreso & $\begin{array}{l}\text { Ley General de } \\
\text { Salud }\end{array}$ & $\begin{array}{l}\text { Cap. } 3 \text { Art.89 } \\
\text { Art.91 }\end{array}$ & $\begin{array}{l}\text { Formación, capacitación y actualización del } \\
\text { personal de salud. } \\
\text { Requisitos para apertura y funcionamiento de } \\
\text { instituciones que formen personal de salud. }\end{array}$ \\
\hline CIFRHS $^{1}$ & $\begin{array}{l}\text { Evaluación de } \\
\text { programas }\end{array}$ & 2013 & $\begin{array}{l}\text { Criterios para evaluar planes y programas de } \\
\text { estudios para la carrera de enfermería }\end{array}$ \\
\hline \multicolumn{4}{|c|}{ Regulación orientada al ingreso al mercado } \\
\hline Actores & Ley & Cap./ Art. & Contenido \\
\hline Congreso & $\begin{array}{l}\text { Ley General de } \\
\text { Salud }\end{array}$ & $\begin{array}{l}\text { Título } 4 . \\
\text { Cap. } 1 \text { Art.79 }\end{array}$ & $\begin{array}{l}\text { Requisito del título para el ejercicio de } \\
\text { actividades profesionales. }\end{array}$ \\
\hline & & Cap. 2 Art. 84 & Prestación del servicio social \\
\hline \multicolumn{4}{|c|}{ Regulación orientada al campo asistencial de la enfermería (control de la competencia) } \\
\hline Actores & Documento & Año & Contenido \\
\hline $\mathrm{SSA}^{2} / \mathrm{CIFRHS}$ & $\begin{array}{l}\text { Lineamientos para } \\
\text { la mejora continua } \\
\text { de servicios de } \\
\text { enfermería }\end{array}$ & 2004 & $\begin{array}{l}\text { Propone metodologías y lineamientos que } \\
\text { permitan mejorar la calidad de los servicios de } \\
\text { enfermería. } \\
\text { Formulación de políticas de calidad } \\
\text { (estándares) }\end{array}$ \\
\hline Actores & Ley/Norma & Año & \begin{tabular}{|c|} 
Contenido \\
\end{tabular} \\
\hline Congreso $\mathrm{CPE}^{3}$ & $\begin{array}{l}\text { Art. } 28 \text { bis de la Ley } \\
\text { General de Salud }\end{array}$ & 2016 & $\begin{array}{l}\text { Lineamientos para la prescripción de } \\
\text { medicamentos por licenciados en enfermería. }\end{array}$ \\
\hline SSA & Acuerdo & 2017 & $\begin{array}{l}\text { Acuerdo por el que se emiten los lineamientos } \\
\text { que contienen procedimientos y criterios } \\
\text { para la prescripción de medicamentos por el } \\
\text { personal de enfermería. }\end{array}$ \\
\hline \multirow[t]{6}{*}{ Congreso } & $\begin{array}{l}\text { NOM-007- } \\
\text { SSA2-2016 }\end{array}$ & $\begin{array}{l}\text { Modificada en } \\
2016\end{array}$ & $\begin{array}{l}\text { Para la atención de la mujer durante el } \\
\text { embarazo, parto y puerperio, y de la persona } \\
\text { recién nacida }\end{array}$ \\
\hline & $\begin{array}{l}\text { NOM-019- } \\
\text { SSA3-2013 }\end{array}$ & 2013 & $\begin{array}{l}\text { Establece lineamientos para la práctica de } \\
\text { enfermería en el Sistema Nacional de Salud. }\end{array}$ \\
\hline & $\begin{array}{l}\text { NOM-022- } \\
\text { SSA3-2012² }\end{array}$ & 2012 & $\begin{array}{l}\text { Condiciones para la administración de la } \\
\text { terapia de infusión. }\end{array}$ \\
\hline & $\begin{array}{l}\text { PROY-NOM-018- } \\
\text { SSA3-20094 }\end{array}$ & 2007 & $\begin{array}{l}\text { Establecer los criterios para la prestación } \\
\text { de servicios de enfermería domiciliarios y } \\
\text { hospitalarios que se contratan y ofrecen a } \\
\text { través de particulares }\end{array}$ \\
\hline & $\begin{array}{l}\text { PROY-NOM-020- } \\
\text { SSA3-20094 }\end{array}$ & 2007 & $\begin{array}{l}\text { Establece lineamientos para la práctica de la } \\
\text { enfermera obstetra en el Sistema Nacional de } \\
\text { Salud. }\end{array}$ \\
\hline & $\begin{array}{l}\text { PROY-NOM-021- } \\
\text { SSA3-2007 }\end{array}$ & 2007 & $\begin{array}{l}\text { Establece lineamientos para la atención } \\
\text { ambulatoria de enfermería a pacientes con } \\
\text { padecimientos crónico-degenerativos y de } \\
\text { tratamiento prolongado. }\end{array}$ \\
\hline
\end{tabular}

para desempeñarse en el campo de interés según instituciones evaluadoras, lo que equivale a reingresar a un nicho más especializado del mercado. En este proceso participan enfermeras organiza- das en colegios autorizados por el Estado para llevar a cabo dicha función.

E2(S): Alguien puede tener el título, su cédula profesional, pero si además tiene una certificación 
Cuadro 1. Documentos orientados hacia la regulación de la enfermería en México.

\begin{tabular}{|l|l|l|l|}
\hline \multicolumn{4}{|c|}{ Formas de contratación (estructura del mercado laboral) } \\
\hline \multicolumn{1}{|c|}{ Actores } & Documento/ Ley & \multicolumn{1}{c|}{$\begin{array}{c}\text { Año de } \\
\text { actualización }\end{array}$} & \multicolumn{1}{c|}{ Contenido } \\
\hline Congreso & $\begin{array}{l}\text { Ley Federal de } \\
\text { trabajo }\end{array}$ & 2015 & $\begin{array}{l}\text { Establece las bases de la relación laboral entre } \\
\text { empleado y empleadores }\end{array}$ \\
\hline $\begin{array}{l}\text { Directivos de } \\
\text { la institución y } \\
\text { miembros del } \\
\text { sindicato }\end{array}$ & $\begin{array}{l}\text { Condiciones } \\
\text { generales de trabajo } \\
\text { de la Secretaría de } \\
\text { Salud }\end{array}$ & $2016-2019$ & $\begin{array}{l}\text { Regula el ingreso, permanencia, baja, cese, } \\
\text { promoción y estímulos de los trabajadores de la } \\
\text { Secretaría de Salud. }\end{array}$ \\
\hline \multicolumn{1}{|c|}{ Actores } & $\begin{array}{l}\text { Contrato colectivo } \\
\text { del IMSS }\end{array}$ & $2017-2019$ & $\begin{array}{l}\text { Regula las relaciones de trabajo entre los } \\
\text { empleados y empleadores. }\end{array}$ \\
\hline $\begin{array}{l}\text { Directivos de } \\
\text { la institución y } \\
\text { miembros del } \\
\text { sindicato }\end{array}$ & $\begin{array}{l}\text { Tabulador de pagos } \\
\text { del IMSS para } \\
\text { enfermeras }\end{array}$ & Mecanismos de pago \\
\hline actualización & $2017-2018$ & Contenido \\
\hline Sabulador de la SSA & Salarios establecidos para el periodo \\
\hline para enfermeras & 2018 & Salarios establecidos para el periodo \\
\hline
\end{tabular}

${ }^{1}$ Comisión Interinstitucional para la Formación de Recursos Humanos para la Salud. ${ }^{2}$ Secretaría de Salud. ${ }^{3}$ Comisión Permanente de Enfermería. Dependencia de la Secretaría de Salud, encargada de conducir y analizar acciones y actividades consideradas de la competencia de enfermería. ${ }^{4}$ Proyectos de norma que se encuentran en proceso de ser aprobados (inscritos a partir del 2007).

eso le debe dar un plus para optar por una plaza de... nivel $C$, nivel $D$ [posiciones con mayor ingreso], o sea, sí tiene un valor también en la dinámica del empleo [...] pero estamos apenas en etapas incipientes en México.

\section{Control de la competencia profesional}

Los actores destacados en este proceso son: la Comisión Permanente de Enfermería (CPE), quien participa en la presentación de proyectos de norma y en su seguimiento, asimismo, el Congreso de la República, encargado de revisar y aprobar las normas propuestas.

En esta dimensión, la regulación de la enfermería es escasa y claramente orientada al ejercicio asistencial institucionalizado. Existen Normas Oficiales Mexicanas (NOM) que establecen el campo de acción de la enfermería en el territorio nacional. Asimismo, existen proyectos de norma (PROY-NOM) que pretenden crear espacios de ejercicio independiente, que aún no han sido aprobados y que permanecen en espera de ser revisados.

A través de las normas aprobadas se observa mayor énfasis en criterios de competencia inter- na (entre los diferentes niveles de formación). La NOM-019 ${ }^{17}$ establece funciones diferenciadas para cada nivel de formación privilegiando el personal de mayor nivel académico. En cuanto a criterios de competencia externa se destaca la NOM- $007^{18}$ y el Art. 28 Bis de la Ley General de Salud (LGS) ${ }^{19}$, ambos establecen la participación de enfermeras con nivel de licenciatura equiparando su responsabilidad y el respaldo de sus actividades con el de otros profesionales de la salud.

En la práctica, el área que las enfermeras han establecido como su espacio natural es la de "los cuidados" la cual es sumamente indefinida ya que incluye acciones de promoción, prevención, atención personal, rehabilitación y paliación. Son diversos los grupos ocupacionales que disputan a la enfermería la ejecución de acciones, particularmente los médicos. En la actualidad un campo específico donde la enfermería ha ganado terreno para establecer un propio modelo de práctica es en la atención del parto de bajo riesgo.

E3(C): Debe haber cambios importantes en la Ley General de Salud [...] para que las enfermeras puedan ampliar su rol [...].

E3(C): Se trabaja el proyecto de Norma-020 para la práctica de la enfermería obstétrica. Esta- 
blece que [...] la enfermera formada con las competencias en el ámbito de cuidado materno, es un actor que debe insertarse [en la estructura de servicios]...

E10(H): En 2016, se modificó la norma 007 donde se incluye a personal no médico en la atención del parto [...] vamos a poder impulsar [para] que se hable más explícitamente de los servicios de partería profesional.

\section{Estructura del mercado de trabajo}

Los principales actores en este campo son las instituciones de salud, públicas y privadas quienes definen sus requerimientos de enfermeras de acuerdo con sus lineamientos internos. Los principales empleadores de enfermeras son la Secretaría de Salud (SSA) y el Instituto Mexicano del Seguro Social (IMSS). En estas instituciones los sindicatos de las instituciones juegan un papel importante al negociar los términos de la contratación del personal de enfermería de acuerdo con los contratos colectivos. Sin embargo, los puestos de trabajo protegidos por el sindicato se han reducido ostensiblemente y las nuevas formas de contratación son temporales y no incluyen los derechos definidos por la ley laboral mexicana. Las instituciones privadas han incrementado su presencia en los últimos 20 años representando una fuente de demanda cada vez más importante pero con condiciones laborales más flexibles con respecto a las instituciones públicas, particularmente relacionadas con la existencia de contratos sin temporalidad definida.

No existe un proceso regulador general del mercado para establecer equilibrios entre la oferta y demanda de enfermeras por lo que cada institución establece sus propias estrategias para el reclutamiento del personal de enfermería por nivel de entrenamiento. Sin embargo, sí existen reglamentaciones laborales que establecen la forma en la que los contratos deben ser ofrecidos y las prestaciones a las que tienen derecho los trabajadores $^{20}$.

De acuerdo con datos de la Secretaría de Salud, entre 2006 y 2018 las enfermeras licenciadas en el mercado laboral han pasado de representar el $16.6 \%$ del total de enfermeras para ubicarse en el $37.1 \%$ con la implícita reducción de la representación de técnicos y auxiliares en enfermería. No obstante, los técnicos en enfermería para 2018 representaban $47.6 \%$ del mercado, siendo aún mayoría ${ }^{21}$. La tendencia al incremento de enfermeras universitarias también se confirma con datos de la $\mathrm{ENOE}^{22}$.
Por otra parte, el tipo de contratación varía de acuerdo con la institución. En la Secretaría de Salud y en el régimen subsidiado del IMSS (IMSS-Prospera), el personal de enfermería eventual con contrato temporal representan $36 \%$ y $35 \%$ respectivamente. Este tipo de contratación en las instituciones privadas alcanzaba el $21 \% \mathrm{del}$ total de enfermeras contratadas. Las instituciones de seguridad social (IMSS e ISSSTE) a la fecha han logrado mantener su planta de trabajadores con contratos permanentes y sustituir a los fallecidos y retirados con nuevo personal ocupando esas posiciones.

E1(C): Ha aumentado el tipo de contratación eventual y por honorarios [en la SSA], y eso realmente para las enfermeras genera mucha inquietud.

E9(A): A partir del año pasado se jubilaron muchas enfermeras [en esta institución], [...] esas plazas no se han boletinado [ofertado] [...] no se si tenga que ver con la nueva reforma laboral, [...] lo que he escuchado es que van a contratar enfermeras sin prestaciones.

E11(M): Aquí hay dos tipos de contratación: La base [posición permanente sindicalizada] y los interinatos, personas que son contratadas temporalmente para ocupar posiciones de trabajo de personal que ya se jubiló en tanto la base sea ocupada por otra persona; mientras se asigna, ellos tienen un contrato temporal, los contratados como interinos firman contrato cada año pero no generan antigüedad [...].

\section{Mecanismo de pago}

Las enfermeras, laboran predominantemente en instituciones, por lo que la forma más común de percibir ingresos es a través de salarios. De acuerdo con datos de la ENOE, en 2016, el 75\% de las enfermeras con empleo, eran asalariadas, $6.4 \%$ se declaraba dueña de una empresa y $15.3 \%$ trabajador por cuenta propia.

Los salarios son establecidos por la entidad que contrata los servicios, siguiendo comúnmente, políticas nacionales y la aprobación de los presupuestos anuales por parte del Congreso Nacional. La Secretaría de Hacienda y Crédito Público aprueba los códigos laborales y los salarios asociados con éstos, para las instituciones públicas de salud. A partir de estos códigos, cada una de las instituciones establece sus categorías salariales. Como se ha señalado, la mayor parte de las enfermeras en el país trabajan para instituciones públicas y las dos instituciones que incorporan a la mayoría de las enfermeras en sus diferentes 
niveles son la Secretaría de Salud (42\%) y el Instituto Mexicano del Seguro Social (34\%). Ambas representan actores poderosos en la negociación de salarios y formas de pago. Los salarios se negocian periódicamente con los sindicatos de las instituciones.

Tanto la SSA como el IMSS tienen formas distintas de categorizar los niveles de enfermería pero en general se dividen en auxiliares, generales y especialistas. Además están las posiciones de mando denominadas de confianza que no se incluyen en las negociaciones sindicales. Tampoco se incluyen los contratos temporales que en la SSA alcanzan el 36\% de todos los puestos de trabajo y en el IMSS menos del 1\%. Cada nivel tiene asignado un código que a su vez contiene la descripción del puesto y el salario. La estructura salarial es compleja ya que la cantidad fija que recibe un trabajador quincenalmente se compone de un conjunto de rubros de acuerdo con la productividad, el riesgo laboral y la ubicación geográfica, entre otros. En la SSA el salario se compone de tres rubros: a) sueldo base mensual, b) asignación bruta mensual y c) ayuda para gastos de actualización bruta mensual. El salario base mensual se utiliza para calcular la pensión del trabajador a su retiro. El resto de los rubros salariales no se utiliza para establecer el valor de la pensión pero si se descuentan impuestos. El valor de los rubros b) y c) puede alcanzar una proporción del 50\%, dependiendo de la categoría ocupacional.

E9(A): [En esta institución] como 90\% de enfermeras tenemos prestaciones, el otro $10 \%$ restante es el que no tiene base [...] ya al gobierno no le conviene tener enfermeras de base, porque no les paga lo que es, ya no va a pagar jubilaciones, prestaciones etc. Tú, como enfermera de base, tienes todas las prestaciones.

\section{Discusión}

Los resultados mostrados plantean que el modelo o mecanismo de regulación de la enfermería en México se encuentra en un proceso de desarrollo y que sus resultados son aún incipientes. El desarrollo de una estructura regulatoria para enfermería se ha acelerado en las últimas dos décadas como consecuencia de cambios globales en el entrenamiento de profesionales de la salud y la adquisición de competencias demostrables, así como en la reforma de los sistemas de salud que promueven la gestión de los recursos de acuerdo con criterios como el control de costos y el incremento de la productividad ${ }^{23}$.
Estos ajustes de la regulación de la enfermería representan una preocupación en diversos países. Por ejemplo, en Australia la reciente ampliación de posiciones y funciones de las enfermeras en el sistema de salud requiere de una actualización de la regulación para generar un orden articulado entre toda esta gama de funciones ${ }^{24}$. El estudio publicado por Robinson y Griffiths ${ }^{25}$ sobre la regulación de la enfermería de los países de la OCDE hace énfasis en aspectos educativos ya que hasta recientemente el entrenamiento de las enfermeras se transfirió hacia las universidades, sin embargo, aún algunos países se mantienen en los hospitales. Además, la movilidad entre países de la Unión Europea (UE) ha demandado estrategias de armonización de los currículos de formación a fin de garantizar posibilidades de empleo en los países que forman parte de ésta.

Para el estudio de la regulación de la enfermería en México se utilizaron cuatro dimensiones que en conjunto constituyen el modelo regulatorio propuesto por Moran y Wood ${ }^{9}$. Cada una de estas dimensiones muestra avances diferenciales en relación con el modelo general. Estas diferencias se establecen tanto en la participación de los actores como en el desarrollo de los procesos, pero particularmente, en los resultados.

La primera dimensión de ingreso al mercado refleja que los mecanismos reglamentarios están establecidos y son suficientemente específicos. A pesar de que el Estado otorga una licencia, se observa su incapacidad para vigilar que los egresados logren incorporarse al mercado en condición de enfermeras con licencia. Este control tampoco es ejercido por los grupos de representación profesional lo cual favorece el incumplimiento de la normatividad vigente y abre espacios de vulnerabilidad, que son aprovechados por las instituciones empleadoras. Existen al menos dos vías de ingreso al mercado que no cumplen con lo reglamentado: las instituciones privadas contratan temporalmente estudiantes activos y personas con formación incompleta en enfermería para cumplir las funciones de una enfermera con licencia, y la incorporación de estudiantes (servicio social) que terminaron créditos universitarios pero que no han recibido ni grado ni licencia, con el fin de ofrecerles un espacio de práctica durante un año. Estos estudiantes llevan a cabo actividades que corresponden frecuentemente a las de una enfermera titulada.

Como se señaló en la sección de resultados, las funciones de la enfermería se establecen alrededor del cuidado el cual incluye un amplio conjunto de acciones de diverso nivel técnico. Siendo el 
cuidado un campo de acción tan amplio, existen diversos grupos ocupacionales que compiten con la enfermería por éste ${ }^{26}$. Esta característica unida a la escasa regulación en dicha dimensión, convierte en un proceso complejo el establecimiento de normas que definan un claro monopolio de práctica y que permitan a la enfermería protegerlo contra sus competidores. Detrás de este fenómeno está el hecho de que la enfermería tiene diferentes niveles de entrenamiento, por lo tanto, las competencias difieren según el nivel de formación. La formación en el nivel técnico implica el desarrollo de competencias básicas, mientras que la de nivel universitario incluye, además de las competencias clínicas, competencias más complejas como la gestión de servicios de salud y la investigación ${ }^{27}$. Sin embargo, un campo de práctica donde se ha avanzado en el control de la competencia es en la atención del parto. A partir de la aprobación de la versión modificada de la norma NOM-007-SSA2 de 2016, se han empezado a implementar modelos de parto respetado donde las enfermeras obstetras han ganado autonomía, capacidad de control sobre su práctica y especificidad en su ejecución ${ }^{28}$. Otras áreas de control de la práctica son más específicas como es el caso de la atención en casa y el tratamiento de heridas.

El mercado laboral de la enfermería muestra diferencias claramente asociadas a la segmentación del sistema de salud mexicano. Se observan tres grandes segmentos, la seguridad social, la Secretaría de Salud y las instituciones privadas. La seguridad social ha orientado sus requerimientos de personal de enfermería al reclutamiento de técnicas a pesar de la disponibilidad cada vez más amplia de enfermeras con licenciatura. El sector privado contrata también preferentemente técnicas en enfermería para ubicarlas en unidades hospitalarias ${ }^{21}$. En cambio, la Secretaría de Salud ha optado por reclutar enfermeras licenciadas conforme se encuentran disponibles en el mercado. Una tercera parte de estas enfermeras está ubicada en unidades de primer nivel de atención. Sin embargo, las condiciones de empleo entre las enfermeras del sector privado y las del sector público se han deteriorado ostensiblemente en la última década a través del fenómeno de precarización ${ }^{29}$. La regulación de estos fenómenos en sus aspectos más esenciales corresponde a las instituciones de salud y a sus estructuras sindicales. A pesar de que la legislación actual -aprobada por la Secretaría de Salud como responsable de la gobernanza del sistema- establece las diferencias entre los niveles de entrenamiento, las competencias y funciones de cada nivel, las instituciones llevan a cabo el reclutamiento a partir de sus propias prioridades. La adecuada ubicación de los niveles de enfermería en la estructura de servicios de salud no es un asunto estrictamente nacional. De hecho, entre los países de la OCDE existen diferencias entre los países que forman enfermeras en universidades y aquellas que los hacen en hospitales con niveles técnicos ${ }^{30}$.

El planteamiento original de Moran y Wood ${ }^{9}$ diseñado para el estudio de la regulación de los médicos hace énfasis en los diferentes mecanismos de pago que existen para ese grupo ocupacional. Sin embargo, en México como en muchos otros países, las enfermeras son preferentemente asalariadas en instituciones públicas. Por lo tanto otros mecanismos de pago como honorarios, capitación o pago por desempeño son sumamente raros en estas instituciones. A pesar de esta homogeneidad, para el caso estudiado fue posible identificar diferencias en las estructuras salariales entre instituciones. Las diferencias salariales para enfermeras que trabajan 40 horas a la semana en instituciones públicas son poco significativas. No obstante, existen diferencias importantes entre trabajadores permanentes y temporales. Los trabajadores permanentes perciben salarios más generosos pero a lo largo del tiempo, éstos se han re-estructurado de acuerdo a una racionalidad que protege a la institución de pagar una pensión por el total del ingreso al final del ciclo laboral del trabajador ${ }^{31}$. En la Secretaría de Salud la disponibilidad de posiciones permanentes se redujo drásticamente al inicio del siglo y la incorporación de enfermeras se realizó a través de contratos temporales. A través de estos contratos el trabajador no tiene derecho a una pensión al final de su ciclo laboral y por lo tanto el salario es compactado, sin diferenciar entre salario base y compensaciones. Esta racionalidad en la estructura salarial es la misma que se usa en el sector privado ${ }^{32}$. La lucha de las enfermeras por el valor de los salarios y las pensiones no es exclusiva de México. En Inglaterra apenas en 2014 se sugirió al Ministerio de Salud un incremento de 1\% para compensar la pérdida del valor salarial de las enfermeras el cual fue rechazado por el gobierno en ese momento pero aplicado hasta 2016 para todo el personal ${ }^{33}$. Otro aspecto que el gobierno inglés ha enfatizado, ha sido sobre la reducción de los contratos terciarizados para el control de $\operatorname{costos}^{34}$.

La regulación es una dimensión del estatus de una profesión. Desde la perspectiva de la sociología de las profesiones, las profesiones buscan garantizar que sus miembros individuales adquieran las capacidades y competencias propias de su 
campo de conocimiento, al más alto nivel, para lo cual deben recibir reconocimientos formales al final del entrenamiento. La regulación también busca que las capacidades adquiridas en el entrenamiento se pongan en juego en el mercado laboral, buscando construir monopolios de práctica a través de excluir de ésta a individuos que no sean capaces de demostrar que cuentan con el entrenamiento correspondiente ${ }^{35}$. Existen otras dimensiones en las profesiones que no son sujetas de regulación, entre las que se encuentran el desarrollo de una ideología profesional o el prestigio social. Por tanto la regulación tiene clara injerencia en el desarrollo de dimensiones concretas de la profesionalización. Un aspecto clave en la profesionalización es que los miembros del grupo ocupacional se unen para convertirse en un actor político que participa -e idealmente controla- la definición de estándares, procesos y resultados de la regulación. En México, el proceso de regulación de la enfermería se ha construido alrededor de la política estatal con la participación creciente de los colectivos de enfermeras. Los resultados apuntan para los próximos años hacia el desarrollo de un modelo de control estatal con participación de la enfermería en aspectos clave.

\section{Conclusiones}

En el aparato regulatorio de la enfermería en México, el Estado es el actor dominante. Sin embargo, recientemente ha fomentado la participación de otros actores, incluidos los representantes de la enfermería. El Estado mantiene bajo su control el licenciamiento como mecanismo de ingreso al mercado. En el mercado también tienen una gran influencia ya que los procesos regulatorios están dominados por las instituciones públicas en las cuales el Estado define políticas de contratación y mecanismos de pago. Como actor principal, el Estado tiene la responsabilidad de generar mecanismos entre la oferta y la demanda que sean compatibles y equilibrados. Por su parte, la enfermería debe desarrollar estrategias para incrementar su participación tanto en el fortalecimiento de la legislación como en la vigilancia de su cumplimiento.

\section{Colaboradores}

La idea, el diseño del trabajo, la recolección de datos fueron realizados por P Aristizabal y G Nigenda. El procesamiento de los datos lo llevó a cabo P Aristizabal. El análisis, la interpretación de los datos y la primera versión del manuscrito, lo realizaron P Aristizabal y G Nigenda. A Squires y Y Rosales revisaron el manuscrito y realizaron aportaciones sustantivas. Todos los autores aprobaron la versión final.

\section{Agradecimientos}

Se agradece al Consejo Nacional de Ciencia y Tecnología (Conacyt) por haber otorgado una beca a la primera autora para financiar sus estudios de doctorado. También se agradece al Instituto $\mathrm{Na}$ cional de Salud Pública por haber brindado un espacio para la formación doctoral de la primera autora. Este artículo es uno de los productos del trabajo de tesis.

\section{Referencias}

1. Nigenda G, Ruiz JA, Rosales Y, Bejarano R. Enfermeras con licenciatura en México: estimación de los niveles de deserción escolar y desperdicio laboral. $\mathrm{Sa}$ lud Publica Mex 2006; 48(1):22-29.

2. Gobierno de México. Secretaría de Salud. México: Sistema de Información y Administración de Recursos Humanos en Enfermería [Internet]. [acessado $2019 \mathrm{Jul}$ 2]. Disponible en: https://bit.ly/32NG0pV

3. Nigenda G, Ruíz J, editores. Formación, empleo y regulación de los recursos humanos para la salud. Bases para su planeación. Cuernavaca Morelos: Instituto Nacional de Salud Pública; 2010.

4. Melo CMM, Carvalho C, Silva A, Leal J, Santos T, Santos H. Força de trabalho da enfermeira em serviços estaduais com gestão direta: Revelando a precarização. Esc Anna Nery [periódico na Internet]. 2016 [acessado 2019 Jul 2]; 20(3):e20160067. Disponible en: http://dx.doi.org/10.5935/1414-8145.20160067

5. Squires AP. A case study of the professionalization of Mexican nursing: 1980 to 2005 [tese]. United States: UMI Microform. Universidad de Yale; 2007.

6. Arakaki J. Significados y concepciones de la Enfermería: el punto de vista de estudiantes de la carrera de la Universidad Nacional de Lanús, 2008-2010. Salud Colectiva 2013; 9(2):151-167. 
7. Organizacíon Mundial de la Salud (OMS). Nursing and midwifery: A Guide to Professional Regulation [Internet]. 2002 [acessado 2018 Jun 2]. Disponible en: https://bit.ly/2JPZeUB

8. Mesa C. Las reformas de salud en América Latina y el Caribe: su impacto en los principios de la seguridad social. Comisión Económica para América Latina y el Caribe. Santiago de Chile: CEPAL; 2005.

9. Moran M, Wood B. States, Regulation and the Medical Profession. Buckingham: Open University Press; 1993.

10. Nigenda G, Machado MH. Modelos de regulación profesional de los médicos en América Latina: elementos teóricos para su análisis. Cad Saude Publica 1997; 13(4):685-692.

11. Sommer-Harrits G. Professional Closure Beyond State Authorization. Professions \& Professionalism [periódico na Internet]. 2014 [acessado 2018 Nov 12]: 4(1):1-17. Disponible en: http://dx.doi.org/10.7577/ pp.567.

12. Yin RK. Case Study Research: Design and Methods. $2^{\text {nd }}$ ed. Thousand Oaks, CA: Sage Publications; 1994.

13. Instituto Nacional de Estadística y Geografia (INEGI) [página na Internet]. [acessado 2019 Maio 12]. Disponible en: https://bit.ly/2SgSCQh

14. Taylor S, Bogdan R. Introducción a los métodos cualitativos de Investigación. Barcelona: Paidós Básica; 1987.

15. Rodríguez G, Gil J, García E. Metodología de la Investigación Cualitativa. 2a ed. Granada (España): Ediciones Aljibe; 1996.

16. Hsieh HF, Shannon SE. Three approaches to qualitative content analysis. Qual Health Res 2005; 15(9): 1277-1288.

17. Norma Oficial Mexicana NOM-019-SSA3-2013. Para la práctica de la enfermería en el Sistema Nacional de Salud. Diario Oficial de la Federación 2013; 2 ago.

18. Norma Oficial Mexicana NOM-007-SSA2-2016. Para la atención de la mujer durante el embarazo, parto y puerperio y de la persona recién nacida. Diario Oficial de la Federación 2016; 7 abr.

19. Acuerdo por el que se emiten lineamientos que contienen el procedimiento y los criterios a los que deberán sujetarse los licenciados en Enfermería, así como los pasantes en servicio social de las carreras referidas en los numerales 1 al 5, del artículo 28 Bis, de la Ley General de Salud, para la prescripción de medicamentos. Diario Oficial de la Federación 2017; 8 mar.

20. México. Ley Federal del Trabajo, de 12 de junio de 2015. Diario Oficial de la Federación 2015; 12 jun.

21. Secretaría de Salud (SSA). Estado de la enfermería en México 2018 [Internet]. México: OPS, OMS; 2018. [acessado 2019 Maio 12]. Disponible en: https://bit. ly/2UDYesB

22. Aristizabal P, Nigenda G, Servan-Mori E. The precarization of the Mexican nursing labour market: A repeated cross-sectional analysis for the period 20052018. Human Resources for Health. In press.

23. Organización Panamericana de la Salud (OPS). Regulación de la Enfermería en América Latina. Washington, D. C: OPS,OMS; 2011. (Serie Recursos Humanos para la Salud No 56).
24. Duffield CM, Gardner G, Chang A, Fry M, Stasa H. National regulation in Australia: A time for standardisation in roles and titles. Collegian 2011; 18(2):45-49.

25. Robinson S, Griffiths P, National Nursing Research Unit. Nursing education and regulation: international profiles and perspectives. London: Kings College London; 2007.

26. Cook LB, Peden A. Finding a Focus for Nursing. The Caring Concept. ANS Adv Nurs Sci 2017; 40(1):12-23.

27. Castañeda-Godínez MC, Pérez-Loredo Díaz L, Pérez-Cabrera I, Müggenburg-Rodríguez Vigil MC. Formación professional de enfermería durante la primera mitad del Siglo XX en la UNAM. Revista Enfermería Universitaria ENEO-UNAM 2010; 7(n. esp.):25-32.

28. Díaz-García S, Zavala-Suárez E, Ramírez-Hernández C. Evaluación de las competencias en enfermería como reflejo de calidad y seguridad en la mujer embarazada. CONAMED 2013; 18(13):104-110.

29. Progianti JM, Moreira NJMP, Prata JA, Vieira MLC, Almeida TA, Vargens OMC. Precarização do trabalho da enfermeira obstétrica. Rev Enfermagem UERJ [periódico na Internet]. 2018 [acessado 2019 mar 13]; 26:e33846. Disponible en: https://doi.org/10.12957/ reuerj.2018.33846

30. Organisation for Economic Co-operation and Development (OCDE). Regions at a Glance. Paris: OECD; 2007.

31. De Lara-Landa M. Derecho al trabajo y trabajo precario en México. Rev Investigación en Derecho, Criminología y Consultoría Jurídica 2017; 11(22):165-190.

32. Sánchez-Ildefonso LA. Análisis de puestos y sueldos de personal de mando en la Administración Pública Federal 2018. Encrucijada 2018; 30:55-76.

33. Department of Health and Social Care. NHS staff to receive $1 \%$ pay rise. Government sets out the pay awards for all NHS staff [reportagem na Internet]. 2016 [acessado 2019 Maio 12]. Disponible en: https:// bit.ly/1U1hCZM

34. BBC News. NHS England cap on agency payments comes into effect [reportagem na Internet]. 2015 [acessado 2019 Jun 12]. Disponible en: https://bbc. in $/ 30 \mathrm{mOUZN}$.

35. Freidson E. La teoría de las profesiones. Estado del arte. Perfiles Educ 2001; 23(93):28-43.

Artigo apresentado em 13/04/2019

Aprovado em 20/08/2019

Versão final apresentada em 30/09/2019 